and bile acid-related metabolites were related to curative effect. The enrichment of Alloprevotella after FMT may be able to treat ulcerative colitis by regulating bile acid metabolism, improving mucosal permeability and regulating immunity. Future work should focus on the multi center verification and the relationship of immunity and gut microbiome.

\section{IDDF2019-ABS-0253 EXPLAINED VARIANCE AND PREDICTABILITY OF INFLAMMATORY BOWEL DISEASES BY GENETIC RISK SCORE IN FIVE ASIAN POPULATIONS (RESULTS FROM THE INTERNATIONAL IBD GENETICS CONSORTIUM)}

${ }^{1}$ Shifteh Abedian* ${ }^{2}$ Sunny H Wong, ${ }^{3}$ Suzanne Van Sommeren, ${ }^{4}$ Atsushi Takahashi, ${ }^{5}$ Jae Hee Cheon, ${ }^{6}$ Ajit sood, ${ }^{7}$ Homayoon Vahedi, ${ }^{8}$ Keiko Yamazaki, ${ }^{5}$ Won Ho Kim, ${ }^{9}$ Thelma BK, ${ }^{10}$ Nasser E Daryani, ${ }^{8}$ Michiaki Kubo, ${ }^{11}$ Suk-Kyun Yang, ${ }^{12}$ Rupa Banerjee, ${ }^{7}$ Reza Malekzadeh, ${ }^{3}$ Rinse K Weersma, ${ }^{2}$ Siew C Ng, ${ }^{1}$ Behrooz Z Alizadeh. ${ }^{1}$ Department of Epidemiology, University of Groningen and University Medical Center Groningen, Groningen, Netherlands; '2Department of Medicine and Therapeutics, Institute of Digestive Disease, State Key Laboratory of Digestive Disease, LKS Institute of Health Science, the Chinese University, Hong Kong; ${ }^{3}$ Department of Gastroenterology and Hepatology, University of Groningen and University Medical Center Groningen, Groningen, Netherlands; ${ }^{4}$ Laboratory for Statistical Analysis, RIKEN, Yokohama, Japan; ${ }^{5}$ Department of Internal Medicine and Institute of Gastroenterology, Yonsei University College of Medicine, Seoul, Korea, South; ${ }^{6}$ Department of Medicine, Dayanand Medical college and Hospital, Ludhiana, India; ${ }^{7}$ Digestive Disease Research Center, Digestive Disease Research Institute, Tehran University of Medical Sciences, Tehran, Iran; ${ }^{8}$ Division of Genomic Epidemiology and Clinical Trials, Clinical Trials Research Center, Nihon University School of Medicine, Tokyo, Japan; ${ }^{9}$ Department of Genetics, University of Delhi South Campus, New Delhi, India; ${ }^{10}$ Department of Gastroenterology, Emam Hospital, Tehran, Iran; ${ }^{11}$ Department of Gastroenterology and Hepatology, Asan Medical Center, University of Ulsan College of Medicine, Seoul, Korea, South; ${ }^{12}$ Department of Gastroenterology ,Asian Institute of Gastroenterology, Hyderabad, India

\subsection{6/gutjn|-2019-IDDFabstracts.211}

Background In the absence of properly designed studies, the clinical implication of genetic findings in Inflammatory Bowel Disease (IBD) is a matter of persistent debate especially in Asian population where the prevalence of IBD including Crohn's Disease (CD) and Ulcerative Colitis (UC) is rising. We aimed to investigate the predictability of IBD, CD, and UC by the means of Genetic Risk Score (GRS), in yet unaffected highrisk individuals from East Asia (EA) and Central Asia (CA).

Methods This present study included 9,698 subjects, consisting of 2,003 CD, 2,730 UC and 4,965 countries, age and gendermatched controls, genotyped on the Immunochip array of three EA (Japan, South-Korea and China) and two CA countries (India and Iran). We generated a multi-locus GRS for each population by combining information from up to 201 known genome-wide significant IBD associated variants to summarize a total load of genetic risk for each phenotype. We estimated explained variance and predictability of IBD, CD, and UC by GRS. We shuffled the EA data into: training set including two out of the three EA populations to build a model to calculate the odds ratio (OR) for each IBD variants, and a test set including the third population for the validation of predictive model built in the training set. For Indian and Iranian populations, we used the previously estimated ORs for the Caucasian population, to build GRS and test the predictive model in these two populations.

Results GRS of IBD could significantly explain up to $4.40 \%$ and $4.14 \%$ of IBD variance in EA and CA populations but given a prevalence of $0.01 \%$ and $0.04 \%$ for IBD it yields to a negligible predictive probability up to $8.8 \times 10^{-4}$ and $5.52 \times 10^{-}$

4. GRS of CD and UC could significantly explain CD and UC to a lesser extent compared to IBD given a lower prevalence of CD and UC (figure1).

Conclusions The present study shows that genetic findings based on Trans-ethnic analyses are applicable across Asian populations. GRS alone can explain a limited percentage of disease occurrence in general population $(<5 \%$ of disease susceptibility) and may not predict IBD in the Asian populations.

\section{IDDF2019-ABS-0254 FACTORS ASSOCIATED WITH READINESS TO SCREEN FOR COLORECTAL CANCER: A POPULATION-BASED STUDY USING THE STAGES OF CHANGE MODEL}

Junjie Huang*, Maggie Chan, Jingxuan Wang, Hanyue Ding, Colette Leung, Martin Wong. Jockey Club School of Public Health and Primary Care, Faculty of Medicine, The Chinese University of Hong Kong, Hong Kong

\subsection{6/gutjnl-2019-IDDFabstracts.212}

Background Colorectal cancer (CRC) screening is crucial in reducing its mortality yet its success depends on long-term participation. To promote the screening programme, there is a need for understanding the factors associated with its uptake. Nevertheless, most previous studies only evaluated the factors associated with screening adherence as a binary outcome. The Stages of Change model (SOC) provides an alternative means to categorise prospective participants into more precise groups. This study aims to investigate the social-demographic factors associated with readiness to CRC screening using the SOC.

Methods A population-based telephone survey was conducted among 2,400 individuals aged 61-70 years old. Data on the social-demographic factors (age, gender, educational level, marital status, occupation, income, smoking and selfperceived health status), past experience; current status; and future intention to receive CRC screening were collected

Abstract IDDF2019-ABS-0253 Figure 1 Odds ratio and explained variance in target populations per phenotype and per state 